\title{
Drugs in the Classroom: Using Pharmacokinetics to Introduce Biomathematical Modeling
}

\author{
G. A. Koch-Noble * \\ Department of Mathematics and Computer Science, Goucher College, 21204, Baltimore, USA
}

\begin{abstract}
Pharmacokinetics is an excellent way to introduce biomathematical modeling at the sophomore level. Students have the opportunity to develop a mathematical model of a biological phenomenon to which they all can relate. Exploring pharmacokinetics takes students through the necessary stages of mathematical modeling: determining the goals of the model, deciphering between the biological aspects to include in the model, defining the assumptions of the model, and finally, building, analyzing, using, and refining the model to answer questions and test hypotheses. Readily accessible data allows students to use the model to test hypotheses that are meaningful to them on an individual level. Students make interdisciplinary connections between this model and their previous personal, mathematical, and other classroom experiences. By beginning with a simple model involving the half-life of a drug, students take advantage of their mathematical abilities to explore the biology. They can then use the new knowledge gained from analyzing the simple model to create more complicated models, thus gaining mathematical and modeling maturity through improving the biological accuracy of the model. Through this experiences, students actually get to $d o$ applied mathematics, and they take ownership of the model.
\end{abstract}

Key words: pharmacokinetics, dynamical systems, biomathematics, math models AMS subject classification: 92-01, 92B99, 92C50, 97M60

${ }^{*}$ Corresponding author. E-mail: gretchen.kochnoble@goucher.edu 


\section{Introduction}

\subsection{Motivation}

In his essay, "Personal Thought on Mature Teaching," David M. Bressoud writes " "Math is not a spectator sport.' We all learn by doing" [3]. He continues with "If you want your students to to be able to apply their knowledge to unfamiliar situations, then give them problems that require this level of understanding" [3]. As we teach our students various topics in mathematics, how often do we give them the opportunity to really participate and do mathematics? Is a problem where friction is neglected really realistic? Are we doing our students a disservice by instructing them to find the right answer? If "we do not...live in a world in which some nebulous other people generate ideas, and hold office, and make decisions," why do we allow our students to learn mathematics in a pre-fabricated, "nice" results, world? [6]

Obviously, students must have some mathematical maturity to explore applied mathematics in a realistic way. But, in the words of Stephen G. Krantz, we "are trying to educate them, to stretch their minds, to teach them to analyze and to think critically" [6]. We all know that there is so much more to mathematics than finding the right answer. There is beauty in the discourse of discovering a new theorem, idea, or model. Why not share that experience with our students?

As a biomathematician, I find that students enjoy exploring a model more when they participate in the entire process: determining the goals of the model, deciphering between the biological aspects to include in the model, defining the assumptions of the model, and finally, building, analyzing, and using the model to answer questions and test hypotheses. Pharmacokinetics is an excellent introduction to mathematical modeling for several reasons. Students can readily discuss the assumptions of the model, as nearly everyone has had experience with taking some medication at some point in their lives. With very little mathematical maturity, students comprehend the concept of a half-life, which is the basis for the initial model. They can build the simplified model from the ground up without having to learn additional mathematics, and the focus of the initial model becomes one of balancing the biological assumptions with the mathematical model. As a result of analyzing the initial model, students develop a more complicated model to more accurately reflect the biology of pharmacokinetics. In other words, they use biology to enhance their mathematical maturity, as they need to understand more complicated mathematical relationships to develop a new model.

Pharmacokinetics is a rich area of research; thus, students can analyze a variety of mathematical models and compare, both quantitatively and qualitatively, the results to known data, as well as comparing and contrasting the different models. Furthermore, students can readily locate data for modeling specific drugs, allowing for many projects, each meaningful to the individual student. If the data is not available in the exact format needed for the model, students learn how to make reasonable assumptions based on the given information. Mathematically, pharmacokinetics combines discrete and continuous equations in a novel way that produces results that students can predict. 
Thus, students learn to judge if the results of the model are reasonable, and they encounter, often for the first time, the realistic situation of not having a single, correct answer. Using the pharmacokinetics model involves students in every level of the mathematical modeling process. They get to $d o$ applied mathematics, and they own the model.

\subsection{Class Structure}

There are two classes in which I use the pharmacokinetics model - a sophomore level "Modeling in the Natural Sciences" course and a junior/senior level "Numerical Methods and Analysis with Applications" course. In the latter, students explore the model as part of a semester-long research project. The focus is more on the students developing the numerical methods to solve the equations in the model and to analyze the model in the context of numerics. In this article, I will focus on using the pharmacokinetics model in the sophomore level class. However, it is important to note that those students who took both courses appreciated the continuity from one course to the next and the ability to analyze the model in a different context.

The sophomore level modeling class is a three-credit course that meets three times a week for fifty minutes. The prerequisite for the course is Calculus II; thus, as one explores various models, it is important to do some "just-in-time" teaching of differential equations and linear algebra. The first time I taught this course, several students were taking our linear algebra class at the same time, and they appreciated seeing the connections between their two classes. As such, one of the lessons I learned is that we can never make the connections between classes too obvious. What is obvious to us, as professional mathematicians, is not always obvious to our students. Depending on each student's Calculus II experience, it may also be necessary to discuss Taylor Series Expansions as a way to move from the continuous to the discrete models. Regardless of the students' preparation, it is necessary to do an in-depth exploration of dynamical systems. This topic serves as a way to connect much of the mathematics that the students have learned at this level to the applications. Finally, the learning objectives for this course are:

1. Students will use their current knowledge and previous experiences to determine what knowledge must be gained to create a mathematical model that accurately reflects the phenomena at hand.

2. Students will pose hypotheses that can feasibly and reasonably be answered via mathematical modeling.

3. Students will create assumptions that reasonably simplify the physical phenomena to make a mathematical model possible.

4. Students will analyze the results, both quantitatively and qualitatively, from the implementation of the mathematical model in both a theoretical manner and in the context of the problem. As part of this analysis, students will compare the results of the mathematical model to known results of experiments. 
5. Students will use the aforementioned analysis to refine the mathematical model to make it more accurate.

6. Students will analyze, discuss, and present peer-reviewed articles that describe mathematical models.

7. Students will determine when to use appropriate technology to implement mathematical models.

8. Students will become interdisciplinary mathematicians by connecting lessons from this class with other mathematics classes, classes in other disciplines, and areas of personal interest.

As I began designing the course, I knew I wanted to approach it in the above manner, and I needed a textbook that would either follow this development or be easily adaptable. I adopted An Invitation to Biomathematics by Raina S. Robeva, et al. [11]. Not only does this textbook explore many biomathematical models, but it does so without requiring students to be experts in either field. There is a laboratory manual that accompanies the text; while I did not use the labs explicitly, I did find this to be a good starting place for asking interesting questions. I supplemented the material from this text with material from texts by Yeargers, et al. and Strogatz [14, 19]. I also found that Neuhauser's Calculus for Biology and Medicine text gave new ways of explaining models and concepts at an accessible level for my students [8]. Finally, the inspiration for the pharmacokinetics model came from an article by Edward Spitznagel entitled Two-Compartment Pharmacokinetics Models, given to me by Professor John Jungck at a BioQUEST Summer Workshop [13].

Assessing the students was based on four components: homework, laboratory exercises, exams, and a paper presentation. Weekly homework assignments came either from the Robeva text or from problems in the Strogatz and Yeargers texts $[11,14,19]$. Exams were take-home exams to allow the students to use the computer to demonstrate their modeling skills; there were also many questions where students had to develop and analyze models on paper. The paper presentation was a short presentation to the class, describing a paper published in a peer-reviewed journal in a field of interest for the student. To assist them, I asked the students to give me their areas of interest, and I found two papers at a level where the majority of the paper would be accessible. Topics included nanotechnology, modeling human choice, and tick modeling, among others. As a result of these presentations, I chose to add a second paper presentation to the course in Fall 2010 to give the students a chance to practice relaying information to the class.

My experiences with the BioQUEST Curriculum Consortium $\left.{ }^{(}\right)$(http://bioquest.org), specifically, the Biological Excel Simulations and Tools in Exploratory, Experiential Mathematics (ESTEEM) Project ${ }^{\circledR}$ (http: / / bioquest.org/esteem), influenced the development of the laboratory exercises. ESTEEM ${ }^{\circledR}$ uses Microsoft ${ }^{\circledR}$ Excel ${ }^{\circledR}$ as a way to implement mathematical models of various natural science phenomena; each implementation comes packaged as a downloadable, adaptable module, and some modules have full documentation and original sources. 
While Excel ${ }^{\circledR}$ does have its limitations when it comes to mathematical modeling, those limitations are greatly outweighed by accessibility (many campuses already have Excel ${ }^{\circledR}$ installed on all campus computers), familiarity (students are not afraid to work with it), and interface (it is easy to change the equations). The ESTEEM ${ }^{\complement}$ project has numerous modules available, for free, under a copy left policy; basically, you may adopt and adapt the modules to fit your needs as long as you do so with proper attribution. The specific topic explored in the laboratory exercises for my class that used ESTEEM ${ }^{\circledR}$ modules were population models, population models with harvesting (a revision of the Continuous Growth Models module), pharmacokinetics, and epidemiology [5, 17, 18]. I developed two laboratory exercises that did not use ESTEEM ${ }^{\complement}$ modules to give students exposure to other ways of implementing mathematical models. One exercise explored various predatory-prey models using the PPlane ${ }^{\complement}$ Java applet, and in another lab, students used Microsoft ${ }^{\circledR}$ Excel ${ }^{\circledR}$ (2007) to analyze genetic inheritance with favorable and unfavorable traits $[10,12]$. Finally, we used Maple ${ }^{\mathrm{TM}} 14$ (Maplesoft) throughout the course as a way to solve complicated problems. Several of the laboratory exercises required students to complete their own research about the model, to make choices based on that research, and to justify those choices. For many students, this was the first time where there was more than one correct answer. As an instructor, these types of labs are challenging from an assessment standpoint. However, one must weigh the benefits that the students received when compared to the challenge one faces while grading the assignments; for me, the benefits win every time.

\section{Pharmacokinetics}

\subsection{Introducing the Model}

Before we even consider any of the mathematics behind pharmacokinetics, I give my class the following definition of pharmacokinetics: "the branch of pharmacology that deals with the absorption, distribution, and elimination of drugs by the body" [7]. With only this description, I ask the class to brainstorm about what they think should be in a mathematical model of pharmacokinetics in a "think/pair/share" method. Some topics that come up with every group: dosage, toxicity, distribution through body, how long it takes the drug to dissolve, how the drug is taken, how big the person is, where the drug has its effect in the body, and how often the drug is taken. Some groups even come up with economic factors such as how hard is it to distribute the drug and more specific chemical ideas such as polarity.

Once we have a master list of topics, we try to group them into similar ideas. We then discuss how feasible it would be to create such a model, and whether such a model would be too specific. In particular, we discuss the idea of "dimensionless models." For many students, and even some of my biology colleagues, anything that is to be relevant as a model must have units attached to it. However, if this is to be the case, then mathematicians would have to create a model for every drug in existence. Rather than try to do this impossible task, we focus instead on the biochemical reactions that can be generalized to most medications. Thus, the only unit that actually goes into the 
model is that of time (hours or days); this is because the Food and Drug Administration requires that all manufacturers publish "how the drug behaves in the body," including the half-life of the drug, before the drug receives approval [16]. For the rest of the model, a "unit dosage" is assumed so that the model can be used for any drug, regardless of whether the dosage is given in milligrams or cubic centimeters.

After creating the list of what is desired to be in the model, a mathematical modeler refocuses on the goals of the model. This allows one to establish those model characteristics that are the most important for an entry-level model. Students readily understand that if one initially tries to make the grandiose model, the probability of becoming hopelessly confused is great. So, as a class we discover that the goals of a pharmacokinetics model are:

1. Determine how much medication is required to achieve the desired effect.

2. Determine how long it will take to reach the desired effect.

The next question that is naturally asked is "How will we know from a model if the desired effect is achieved? We know when we feel better, but how does math tell us that?" Robeva, et al. define the therapeutic window as "the range of concentrations over which the drug is both effective and safe," and this range occurs between the minimum effective concentration and the minimum toxic concentration [11]. Thus, if the concentration of the drug in the body remains in the therapeutic window, we say that it is at steady state in the body. It should be noted that the definition of steady state is different in other mathematical and biological contexts. In this case, it does not matter if the drug concentration in the body remains constant as long as it remains within the therapeutic window, also known as the therapeutic range for the drug [2]. In fact, a good discussion to have with one's class is whether we would ever expect the concentration of a drug to remain constant. Most students will quickly state that we would expect to see the concentration go up and down or oscillate. A natural discussion to have next would be to determine what causes this oscillation; again, a brief "think, pair, share" session will result in the realization that the frequency of taking the medication may not match the rate at which the body eliminates it.

After these discussions, we talk about how to formulate such a model mathematically. The following are the key ideas that need to be included to achieve our goals:

1. The model needs to incorporate how the drug is eliminated from the body; this is related to the half-life of the drug.

2. One usually takes a drug in a discrete manner, i.e. take one pill every four hours. So, the dosing of the drug is a discrete process.

3. The way in which the drug is eliminated from the body is continuous.

4. If we incorporate the half-life of the drug, we are looking at the decay of the drug, or how it changes in time. This is some sort of derivative. 
5. We should consider how the drug moves through the body - how does a pill that we swallow make our headache go away?

In total, this discussion should not take more than thirty minutes, depending on the size of the class. The next step is to introduce the idea of compartment models by creating the simplest pharmacokinetics model: the single injection model.

\subsection{Model 1: The Single Injection Model}

To stimulate the biological ideas behind the model, the class discusses what element in the body must be in every pharmacokinetics model, and quickly determines that the bloodstream, or the "transportation highway" for the body, must be included in every model. One way for a drug to enter the bloodstream directly is through a series of injections. Then, a diagram of a model is shown in Figure 1. However, as a simplification, we assume that only one injection is given. Thus, the diagram of the model becomes Figure 2, and the initial conditions for the model represent the injection. Let $b(t)$ be the amount of the drug in the bloodstream at any time $t$, in hours. Let $\alpha$ be

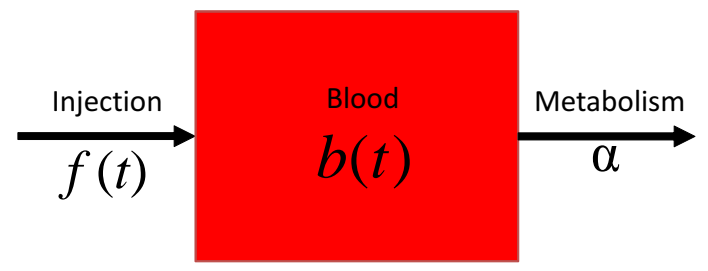

Figure 1: A compartment representation of a multiple injection model.

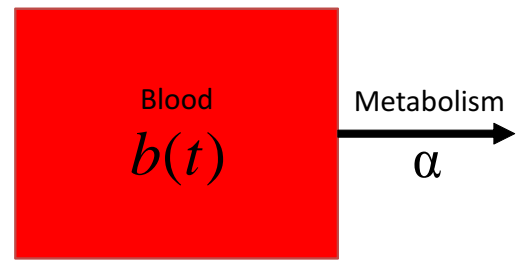

Figure 2: A compartment representation of a single injection model.

the rate at which the drug is metabolized. If we assume only one injection, we know that the initial conditions for this model corresponds to the amount of the drug that is injected; let this amount be $B_{0}$. Then, we have $b(0)=B_{0}$. To determine the equation that governs this system, one must first determine how to create equations based on compartment models.

In general, compartment models describe the transfer of a substance in and out of the compartment, as shown in Figure 3. Simply put, the rate of change of the amount of the substance in the compartment is equal to the difference between the rate in which the substance enters the compartment and the rate at which it leaves. This is a very intuitive concepts for students; however, 
most will not understand it as the Balance Law unless it is explained in the terms above. Furthermore, students will appreciate that the Balance Law basically means that we can neither create nor destroy any matter inside the compartment. Before we formulate any equations, we first need to

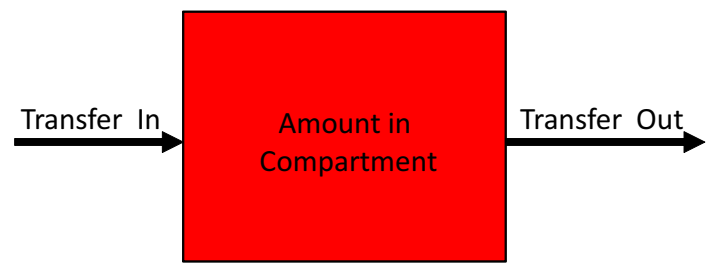

Figure 3: A generic one compartment model.

express the mathematical relationship in words; this is an important skill for our students to learn so they can communicate their ideas - they must be able to move between the mathematical and descriptive expressions seamlessly. Then, we have

Change in amount of substance in compartment $=$ Transfer in - Transfer Out.

Returning to our single injection model (Figure 2), we translate Eq. (2.1) to be

$$
\frac{d b}{d t}=-\alpha b(t)
$$

It is important to discuss the $\alpha b(t)$ term, as students may not readily realize that the rate at which the drug leaves the bloodstream is proportional to the amount in the bloodstream. Then, our system is Eq. (2.2) subject to the initial condition of $b(0)=B_{0}$. Since Eq. (2.2) is a separable differential equation, it is readily solvable by students with a Calculus II background. Then, the solution to the single injection model is

$$
b(t)=B_{0} e^{-\alpha t} .
$$

The next step is important in biomathematical modeling - we must bring the mathematics back to the biology. Recall the desire to include the published half-life of the drug. Then, how do we relate $\alpha$ to the half-life, $\tau$, of the drug? By definition, $b(\tau)=B_{0} / 2$. Then, the following derivation is important for students to see so they may note that $\alpha$ is not the half-life of the drug; rather, it is related to the half-life. The level of detail in this derivation serves a secondary purpose of reviewing the properties of logarithms.

$$
\begin{aligned}
\frac{B_{0}}{2} & =B_{0} e^{-\alpha(\tau)} \\
\frac{1}{2} & =e^{-\alpha(\tau)} \\
\ln (1 / 2) & =(-\alpha)(\tau) \\
-\ln (2) & =(-\alpha)(\tau) \\
\alpha & =\frac{\ln 2}{\tau}
\end{aligned}
$$


Then, if we desire to have the model in terms of the half-life of the drug, we find

$$
b(t)=B_{0}\left(\frac{1}{2}\right)^{t / \tau} .
$$

Eq. (2.4) makes the decay of the drug according to its half-life readily apparent, and the derivation reminds students how to simplify exponential functions.

Deriving the equations is not enough. It is important for students to examine a graphical representation of the model (Figure 4) and to tie that visualization to a verbal statement of what is happening. In this case, the model is a simple decaying exponential function, and the class should discuss why this case is not surprising. A similar mathematical explanation for an initial pharmacokinetics model is given in [11].

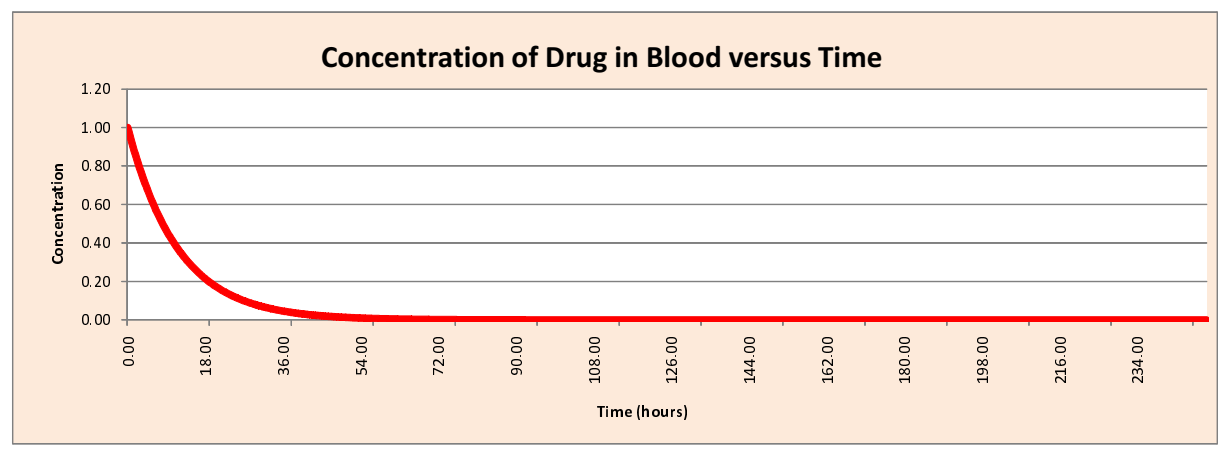

Figure 4: A graph of $b(t)$ versus time for the single injection model.

\subsection{Model 2: The Multiple Injection Model}

A natural extension of the single injection model is to consider what happens if multiple injections are given over a period of time. Consider Figure 1, where $b(t)$ is the amount of the drug in the bloodstream as a function of time, $t$, in hours. Let $f(t)$ describe the dosing pattern of the injections as a function of $t$, in hours. Spitznagel describes $f(t)$ as a discrete pulse function whose width and height depend on the drug being administered [13]. A representation of $f(t)$ is shown in Figure 5. Finally, let $\alpha$ be the metabolism of the drug such that $\alpha=\ln (2) / \tau$, where $\tau$ is the half-life of the drug being modeled. Then, applying the Balance Law, it is clear that

$$
\frac{d b}{d t}=f(t)-\alpha b(t)
$$

This may be one of the first examples of an equation where students see a discrete function as part of the description of a continuous function. More important, some students will realize that the graph of solution to this equation looks just like a series of the graphs of Eq. (2.3) graphed together. In other words, the students recognize that, absent any other input, the amount of the 
drug in the bloodstream will decay exponentially until the next injection is received. At that time, the amount in the bloodstream will spike back up to its initial value, and the cycle will continue, as shown in Figure 6. As seen in Figure 6, there is a slight delay in time until the drug reaches the steady state in the bloodstream (after the fifth dose, around $t=48$ hours). This is a nice illustration of the delay in the drug concentration reaching the therapeutic window.

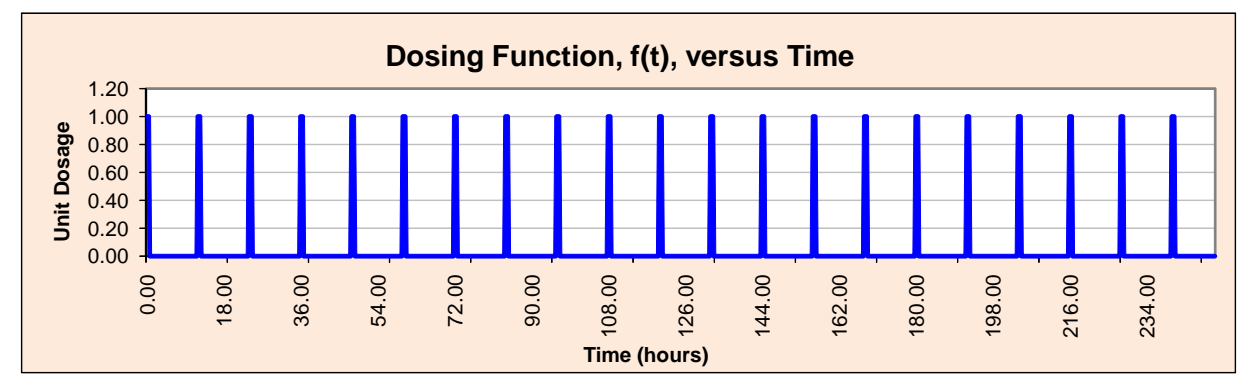

Figure 5: A graph of the dosing function $f(t)$ versus time.

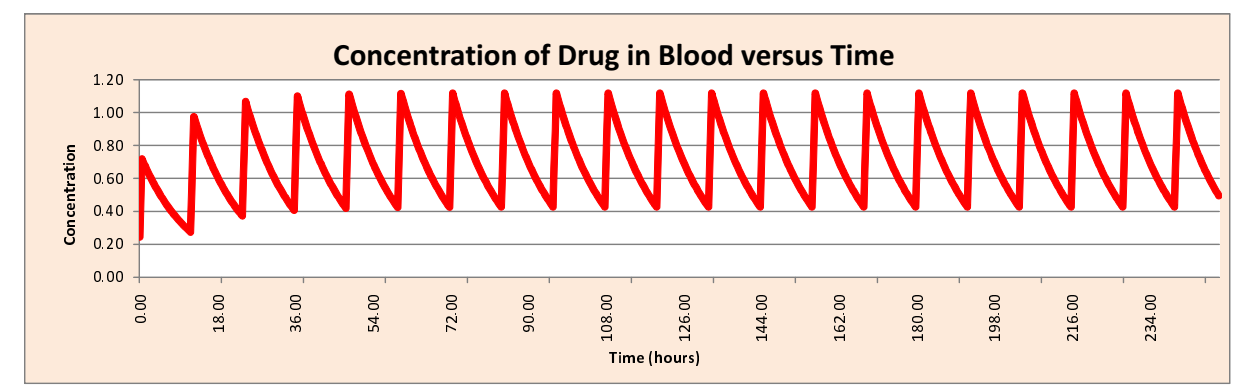

Figure 6: A graph of $b(t)$ versus time for the multiple injection model.

\subsection{Model 3: The Two-Compartment Model}

After exploring the two different versions of an injection model, students are ready to create a more realistic, two-compartment model. As we did with both one-compartment models, we begin by drawing the diagram as shown in Figure 7. Let $f(t), b(t)$, and $\alpha$ be as previously defined. Let $g(t)$ be the amount of the drug in the gastrointestinal tract at any time $t$, in hours, and let $\beta$ represent the metabolism of the drug in the GI tract. Since we are making the assumption that the only way for the drug to move into the bloodstream is from the GI tract, we know that the transfer rate into the bloodstream must be the same as the decay rate in the GI tract; in other words, we cannot destroy the drug in the GI tract without having equal absorption into the bloodstream. This is a theoretical assumption as some drugs are degraded by enzymes as they pass through the intestinal walls [2]. Then, using the Balance Law and the idea that the metabolism of the drug is proportional to the amount of the drug in each compartment, students readily create the 


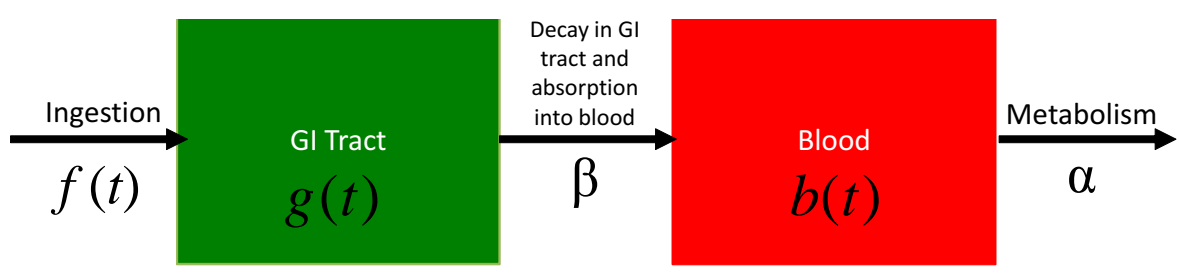

Figure 7: A representation of a two-compartment pharmacokinetics model.

two-compartment, pharmacokinetics model shown in Eq. (2.6).

$$
\begin{aligned}
& \frac{d g}{d t}=f(t)-\beta g(t) \\
& \frac{d b}{d t}=\beta g(t)-\alpha b(t) .
\end{aligned}
$$

While the model is easy to create, the solution is not easy to find since this is a non-homogeneous system. Without a background in differential equations, students would not be able to solve this equation. At this point, depending on your goals for the class, it would be appropriate to explore how to solve a system of homogenous differential equations, given in matrix form, by exploring the solution to Eq. (2.7).

$$
\left[\begin{array}{l}
\dot{g} \\
\dot{b}
\end{array}\right]=\left[\begin{array}{rr}
-\beta & 0 \\
\beta & -\alpha
\end{array}\right]\left[\begin{array}{l}
g \\
b
\end{array}\right]
$$

An excellent reference for such exploration is [14]. By introducing the linear algebra concepts of eigenvalues and eigenvectors that are needed to explore the stability of fixed points in dynamical systems, students connect many different courses together; this is also a nice way to examine epidemiology models, which are the next topic in the class.

The fixed points of the system in Eq. (2.7) occur where

$$
\left[\begin{array}{l}
\dot{g} \\
\dot{b}
\end{array}\right]=\left[\begin{array}{l}
0 \\
0
\end{array}\right] \text {. }
$$

Then, the fixed points are

$$
\left[\begin{array}{l}
g^{*} \\
b^{*}
\end{array}\right]=\left[\begin{array}{l}
0 \\
0
\end{array}\right] .
$$

To determine the stability of the fixed point, we examine the determinant, $\Delta$, and trace, $\tau$, of the matrix in Eq. (2.7). Then, $\Delta=\alpha \beta$, and $\tau=-(\alpha+\beta)$. Then, since $\alpha>0$ and $\beta>0$, we know that $\Delta>0$ and $\tau<0$. This makes the fixed points of the system stable. Whether the fixed point is a stable node or stable spiral will depend on whether $\tau^{2}-4 \Delta$ is positive or negative. Here, $\tau^{2}-4 \Delta=(\alpha-\beta)^{2}$, which is always positive. Thus, the fixed point is a stable node. The theory and methods to support this conclusion can be found in many texts, including [14]. But, this is the 
case where there is no dosing function; so it makes sense that the body will tend towards having no drugs in the system. In order for the students to gain an appreciation of the dynamics of the full pharmacokinetics model, an exploration of the models using visualization techniques proves to be very beneficial.

\section{Exploring the Models}

By diving into the model and exploring the effects that various drugs have, students gain a deeper, and sometimes personal, understanding of the pharmacokinetics model. A guided exploration, in the form of a group laboratory exercise, is one way to accomplish this. To aid students in exploring the model, I created several Excel ${ }^{\circledR}$ modules that allow students to vary the parameters of the model and to explore how the half-life, dosing frequency, and missed doses affect the model. These modules are part of The Biological Excel Simulations and Tools in Exploratory, Experiential Mathematics (ESTEEM) Project ${ }^{\complement}$ (http://bioquest.org/esteem) and are available for adoption and adaption. The first module explores the one-compartment models described in Eq. (2.2) and Eq. (2.5), while the second module models Eq. (2.6) for both a single dose and multiple dose cases. Both modules allow the user to experiment with the effects of missing doses, either one missed dose on a certain day or missing the same dose (i.e. the lunchtime dose of an antibiotic) every day. Finally, there are two versions of each module; one version explores the model over a shorter time period, while the other allows the user to model drugs with half-lives on the order of days. A screenshot of the two-compartment module is shown in Figure 8. The complete pharmacokinetics module can be found at http://bioquest.org/esteem/esteem_details. php?product_id=39111. [5]

Here is a sample laboratory exercise that I created for my students. Students work in groups, are given one class period to begin the lab, and have a week to submit their final write-up.

1. Set the dosing function to zero. Determine the values for $\alpha$ and $\beta$ that give a stable node and a stable spiral, if possible. Be sure to include copies of the graphs with your lab write-up.

2. Read Spitznagels "Two-Compartment Pharmacokinetics Models” [13].

(a) Replicate the results for the usual dosing of PPA and CPM. Describe what happens to the patient.

(b) Change the dosing to be less frequent and more frequent without skipping doses for both PPA and CPM. Describe what happens to the patient, and compare/contrast to results from 2(a).

(c) Using the "Missed Doses" worksheet, create the model where the patient takes the drug as prescribed (i.e. for PPA every 6 hours), but skips every third dose. Describe what happens to the patient, and compare/contrast the results from 2(a) and 2(b). 


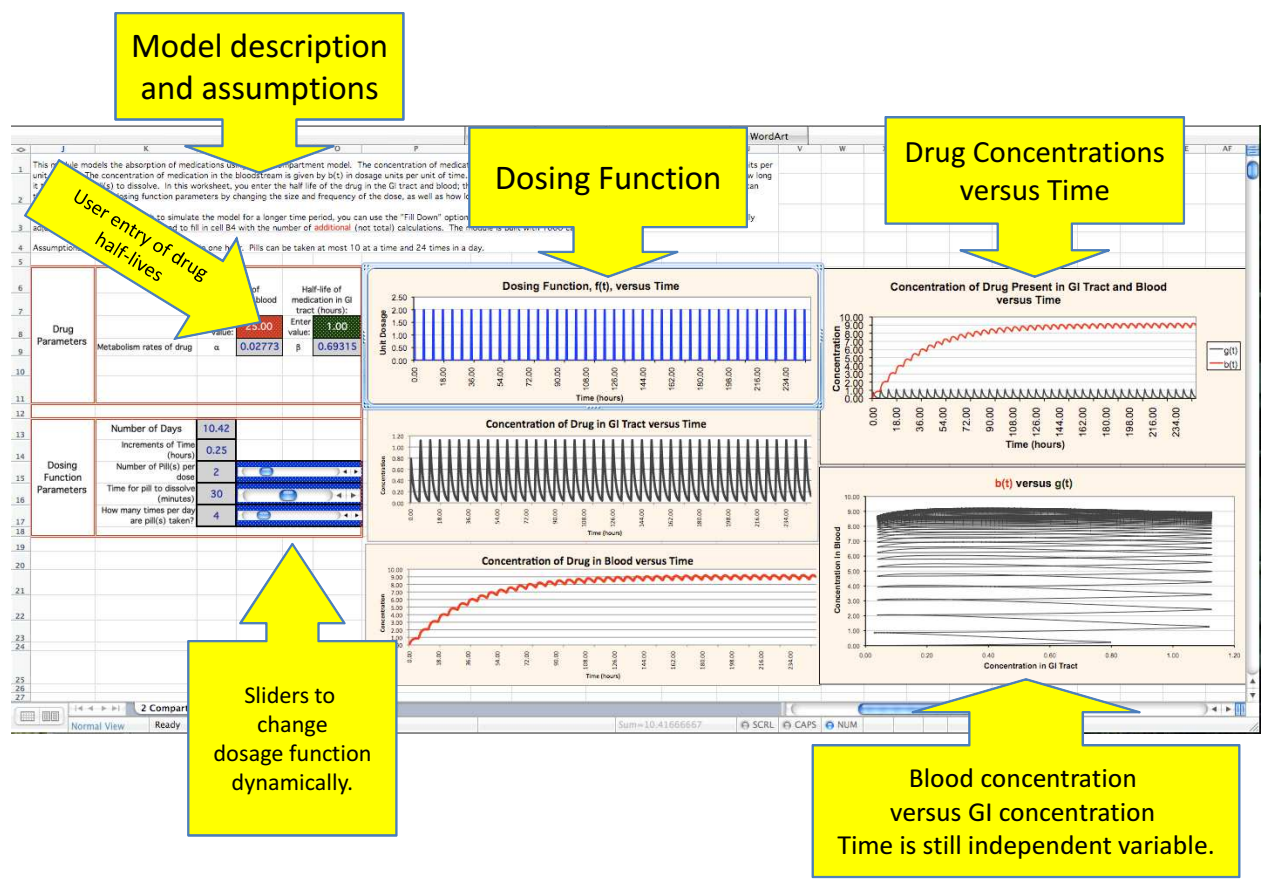

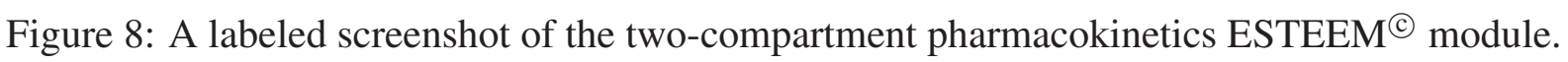

3. Using your research skills, find some real-world drug data. For each drug, repeat the steps of 2(a)-2(c). Note that you will need to use caution with what module goes with which drug. Make sure your units are consistent!

(a) Lithium

(b) Caffeine

(c) Synthetic thyroid hormone (brand name is Synthroid $\cap$ )

(d) Aygestin $\mathbb{R}$

(e) Depo-Provera ${ }^{\mathrm{TM}}$

(f) A drug from the grab-bag of drug inserts.

(g) A drug of your choice.

As is the case with all exploratory exercises in this class, the first problem involves recreating some known results. This gives them the opportunity to familiarize themselves with the modules and extracting the parameters to input into the module with the safety net of being able to check their answers. Students are also reading an original research article and having to extract information from it; this is a valuable skill that can only be learned by doing. It is also important for students to bring the mathematics back to the application and answer questions in context of the model. By having them research the half-lives of various drugs, they will learn how to sort through mounds of information to get the parameters they desire. By having a variety of drug inserts (collected from 
colleagues, friends, pharmacists, and family members), students literally hold the information in their hands, and we can work together to decipher the inserts. This allows them to be more efficient sorting through the data online. If students are stuck for where to go for valid information, the Food and Drug Administration requires that all manufacturers publish the half-life of medications. This information can be found by typing in the drug's name at http://www . accessdata. fda . gov/scripts/cder/drugsatfda/index. cfm and selecting the "Label Information" link [15]. Allow the students to try to find their own sources, but do not let them become so frustrated that they give up on the whole exercise. It is important to note that, in general, only the half-life of the drug in the bloodstream is published. To determine the half-life of the drug in the gastrointestinal tract, one must infer the half-life from the dosing directions. One must also infer how long it take the drug to dissolve. To input all of the known information into the module, the user need only type in the half-lives; all other parameters can be adjusted using sliders, allowing one to see the model change dynamically as the parameters change, as shown in Figure 8.

The particular drugs were selected to highlight different aspects of the pharmacokinetics modules. Lithium is an interesting drug because its dosing must be closely monitored as it has a narrow therapeutic window [13]. Caffeine is the one of the most abused drugs, and many mathematicians, including myself, are guilty of using coffee to fuel our work. This is a drug that is not often thought of as a medication, but by exploring the effects of caffeine, students begin to see other applications for the pharmacokinetics model. Synthroid $R$ is a drug that has a long half-life and takes many weeks to achieve a steady state [1]. Finally, Aygestin $\mathbb{R}$ and Depo-Provera ${ }^{\mathrm{TM}}$ are both forms of progesterone birth control; however, the former is an oral form of birth control, while the latter is injected $[4,9]$.

\subsection{Example Laboratory Results}

Aygestin $\mathbb{R}$ has an average half-life of 8.51 hours in the blood, is supplied in $5 \mathrm{mg}$ tablets, and since there are no specific dosing directions given, especially with respect to food, it is reasonable to assume a half-life of 30 minutes in the gastrointestinal tract and dissolves within 15 minutes [4]. Inputting this information into the one-compartment pharmacokinetics module, we obtain Figure 9. Under these conditions, Figure 9 shows that a steady state is achieved on the third day; if we interpret the steady state to be in the therapeutic window, this model implies that the drug is effective after three days. (Note: the results of this model should never be taken in place of a licensed physician's advice.)

One of the dangers of taking birth control pills is missing a dose. A natural question to ask is what kind of effect missing a dose of Aygestin $\AA$ will have on the steady state of the drug in the bloodstream. To answer this question, suppose the patient forgets to take her pill on the 6th day. Figure 10 shows the results of the simulation. Clearly, this single missed dose has a significant impact on the amount of the drug in the bloodstream staying within the therapeutic window. If this missed dose should correspond in the right way to the woman's hormonal cycle, a pregnancy could result. 
Depo-Provera ${ }^{\mathrm{TM}}$ has an average half-life of 43 days and is administered as a single shot, once every three months [9]. Using the one-compartment, single injection model, we obtain the results shown in Figure 11. From the results, we can gain an understanding of when the drug is no longer effective. Since the shot must be given every three months, we can infer from the model that the drug is not effective after approximately $70 \%$ has decayed. Since we know the explicit solution to this model, we could find the exact percent decay that leaves the drug ineffective.

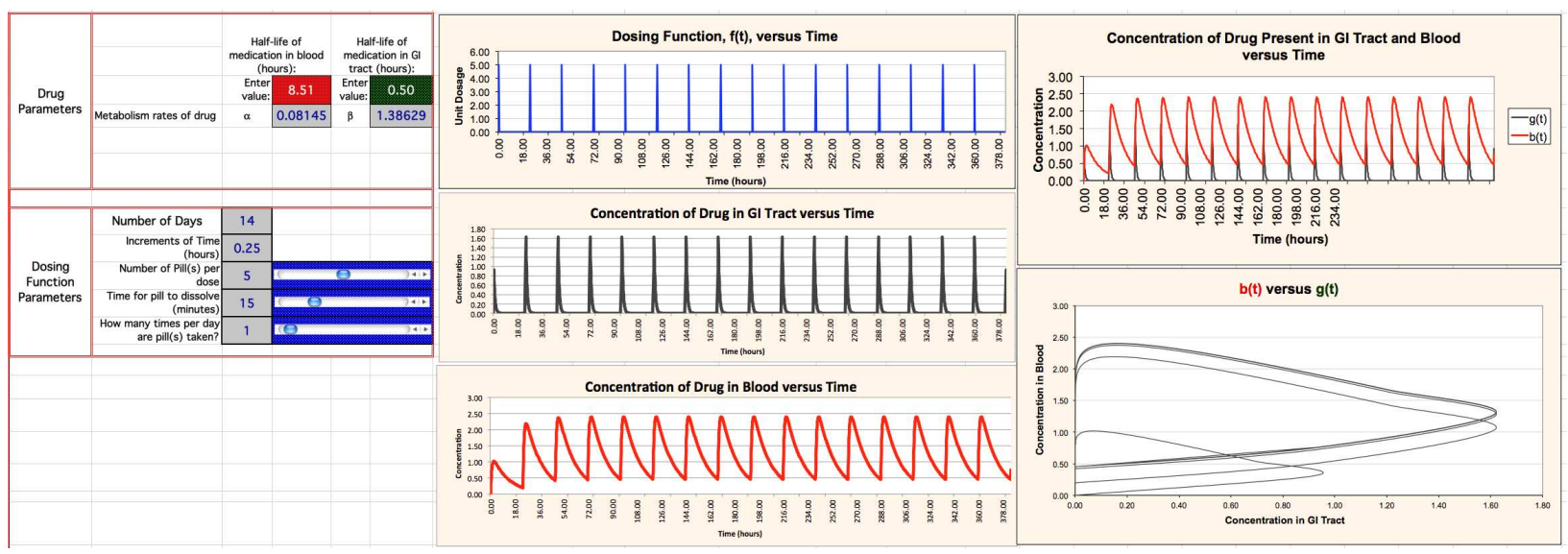

Figure 9: Aygestin $®$ pharmacokinetics model results for a two week period

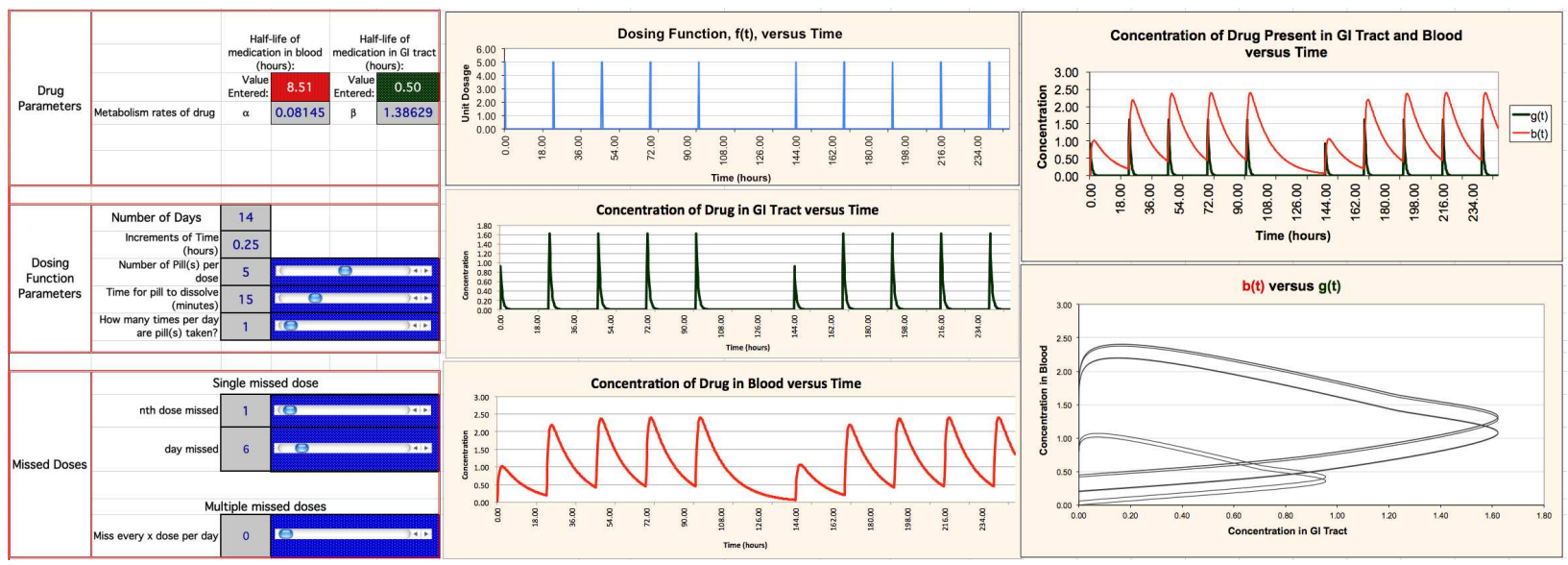

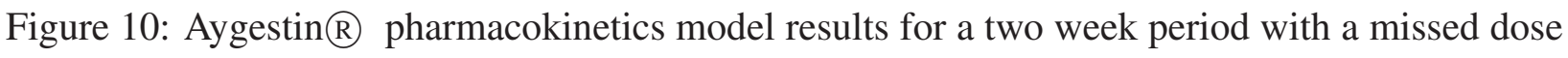
on day 6 


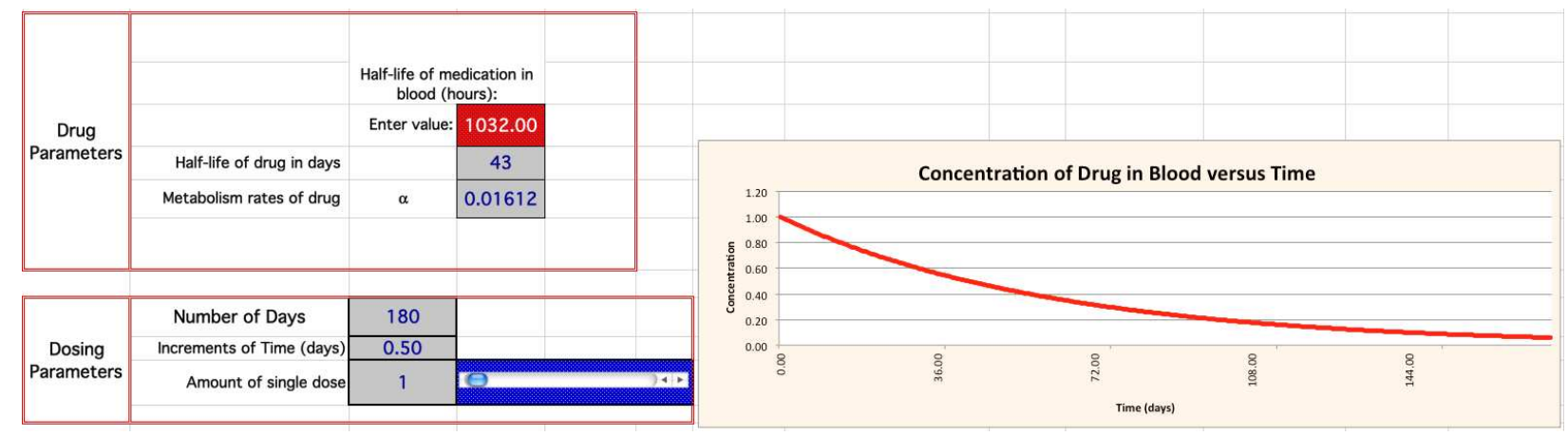

Figure 11: Depo-Provera ${ }^{\mathrm{TM}}$ pharmacokinetics model results for a six month period

\subsection{Conclusion}

This exercise can be expanded beyond this class. By continuing to revisit this model throughout the mathematics curriculum, we provide our students with a continuity that allows them to make the crucial connections throughout the discipline. Some questions that can be explored in other classes are

- How do we get from the half-life to the rate of decay with no numbers?

- What other models use a similar differential equation system?

- How does having a non-constant dosage function affect the analytical solutions?

- What are the analytical solutions for the non-homogeneous system?

- How do we solve this system of equations numerically?

There are several common themes that recur in this course, and it is important to emphasize these themes to help students make the connections. Some of the themes are

- Compartment models (Balance Law, differential equations),

- Discrete versus continuous (Taylor Series Expansions, numerical methods),

- Dynamical systems (bifurcations, chaos, discrete versus continuous analyses), and

- Systems of ordinary differential equations (analytical and numerical solutions, linear algebra).

Overall, this exercise is well-received by the students. Students often have a difficult time sorting through the drug inserts and are quick to note that it would be very difficult for someone with no background in pharmacokinetics to even begin to understand the insert. There may be too much freedom in allowing students to select a drug of choice, as students may choose to model recreational drugs. It is more important to me to have the lab be meaningful to my students; if they 
get to model a drug of choice, they own that model. Since my students from my class two years ago still talk about this lab exercise, the experience of the lab must have made a great impact on my students.

\section{Acknowledgements}

The author is grateful to the following people and institutions: Dr. John Jungck, Beloit College; Dr. Anton Weisstein, Truman State University; Dr. Ethel Stanley, Beloit College; Dr. Sam Donovan, University of Pittsburgh; Dr. Claudia Neuhauser, U. Minn. Rochester; Dr. Marsha Timmerman, LaSalle University; Tracey Schatteman, Lincoln Land Community College; past participants of the BioQUEST Summer Workshops for their input; Goucher College Faculty Affairs Committee grants for travel support; the Nancy Larrick Crosby '30 Fund for Excellence in Teaching Fund for funding the development of the two courses; Goucher College; BioQUEST Curriculum Consortium; the reviewers for their feedback; my departmental colleagues for supporting the development of this course, and my students in MA 216 in Spring 2009 for their input and candid feedback.

\section{References}

[1] Abbott Laboratories. Synthroid $R$ Product Insert/Physician Information. http://www . accessdata.fda.gov/drugsatfda_docs/label/2008/021402s017lbl. pdf (accessed 11 October 2010). North Chicago, 2008.

[2] Anonymous reviewer comments, received December 2010.

[3] D. M. Bressoud. "Personal Thoughts on Mature Teaching." In How to Teach Mathematics by S.G. Krantz. 2nd ed., 173-181. American Mathematical Society, Providence, 1999.

[4] Duramed Pharm., Inc. Aygestin $₫$ Product Insert/Physician Information. http: //www . accessdata.fda.gov/drugsatfda_docs/label/2007/018405s0231bl. pdf (accessed 11 October 2010). Pomona, 2007.

[5] G. A. Koch. Pharmacokinetics. A module of the Biological ESTEEM Collection, published by the BioQUEST Curriculum Consortium. http://bioquest.org/esteem/ esteem_details.php?product_id=39111 (accessed 23 September 2011). 2011.

[6] S. G. Krantz. How to Teach Mathematics. 2nd ed. American Mathematical Society, Providence, 1999.

[7] V. Neufeldt \& D. B. Guralnik (ed). Webster's New World College Dictionary. 3rd ed. Macmillian, New York, 1997.

[8] C. Neuhauser. Calculus for Biology and Medicine. 2nd ed. Pearson Education, Upper Saddle River, 2004. 
[9] Pharmacia \& Upjohn Company. depo-subQ provera $104^{\mathrm{TM}}$ Product Insert/Physician Information. http://www.accessdata.fda.gov/drugsatfda_docs/label/ 2009/021583s0111bl.pdf (accessed 11 October 2010). New York, 2009.

[10] J. C. Polking. PPLANE 2005.10. http://math.rice.edu/ dfield/dfpp.html (accessed 10 October 2010). 1994-2005.

[11] R. S. Robeva, et al. An Invitation to Biomathematics. Academic Press (Elsevier), Burlington, 2008.

[12] R. S. Robeva, et al. Laboratory Manual of Biomathematics. Academic Press (Elsevier), Burlington, 2008.

[13] E. Spitznagel. Two-Compartment Pharmacokinetics Models. C-ODE-E. Harvey Mudd College, Fall 1992.

[14] S. H. Strogatz. Nonlinear Dynamics and Chaos with Applications to Physics, Biology, Chemistry, and Engineering. Addison-Wesley, Reading, 1994.

[15] U. S. Food and Drug Administration. Drugs@FDA. http: //www.accessdata.fda . gov/scripts/cder/drugsatfda/index. cfm (accessed 11 October 2010). 2010.

[16] U. S. Food and Drug Administration. New Drug Application. http: / /www.fda.gov/Drugs/DevelopmentApprovalprocess / HowDrugsareDevelopedandApproved/ApprovalApplications / NewDrugApplicationNDA/default.htm (accessed 10 October 2010). 2010.

[17] A. E. Weisstein. Continuous Growth Models. A module of the Biological ESTEEM Collection, published by the BioQUEST Curriculum Consortium. http://bioquest.org/ esteem/esteem_details.php?product_id=197 (accessed 10 October 2010). 2005.

[18] A. E. Weisstein. SIR Model. A module of the Biological ESTEEM Project, published by the BioQUEST Curriculum Consortium. http://bioquest.org/esteem/esteem details.php?product_id=196 (accessed 10 October 2010). 2003.

[19] E. K. Yeargers. An Introduction to the Mathematics of Biology: With Computer Algebra Models. Birkhäuser, Boston, 1996. 